\title{
Políticas públicas en latinoamérica de lucha contra \\ la violencia de la mujer
}

Autores:

Idme Condori, Walter

widme@ucvvirtual.edu.pe

Ferro Vásquez Arturo

aferro@ucvvirtual.edu.pe

Escalante Gutiérrez Jenner Benjamín

jescalante@ucvvirtual.edu.pe

\section{RESUMEN}

Objetivo: describir y caracterizar las políticas públicas de lucha contra la violencia de la mujer en Latinoamérica y su evolución. El enfoque metodológico que se desarrolla es el descriptivo, se empleó el método de análisis documental en base a la revisión documental de artículos de revistas indexadas como Scopus y Scielo. La investigación llego a la conclusión de que las políticas públicas comprenden un proceso complejo de entendimiento y tratamiento por ello es necesario la aplicación de un modelo integral que contemple factores políticos, culturales, económicos y de salud. Con el propósito de erradicar la violencia contra la mujer, es importante que para aplicar políticas públicas todos los actores y participantes colaboren.

Palabras clave: políticas públicas; estado; violencia de la mujer. 


\title{
Public policies in latin america to combat violence against women
}

\begin{abstract}
Objective: to describe and characterize public policies to combat violence against women in Latin America and its evolution. The methodological approach that is developed is descriptive, the method of documentary analysis was used based on the documentary review of articles from indexed journals such as Scopus and Scielo. The research came to the conclusion that public policies comprise a complex process of understanding and treatment, therefore it is necessary to apply a comprehensive model that takes into account political, cultural, economic and health factors. In order to eradicate violence against women, it is important that all actors and participants collaborate to apply public policies.
\end{abstract}

Keywords: public policies; state; violence of women.

Artículo recibido: 30 noviembre. 2021 Aceptado para publicación: 29 diciembre 2021 Correspondencia: ycuevaro@ucvvirtual.edu.pe Conflictos de Interés: Ninguna que declarar 


\section{INTRODUCCIÓN}

La violencia contra las Mujeres origina una gran preocupación y alarma a nivel nacional e internacional por los índices de incrementos de casos de mujeres violentadas y por las afecciones presentadas al ámbito familiar y al desarrollo integral de los niños (Comité Estadístico Interinstitucional de la Criminalidad, CEIC, 2021).

La Organización Mundial de Salud (2021) y Signorelli et al. (2018), refiere que alrededor de 736 millones de mujeres sufren violencia; el equivalente a afirmar que 1 de cada 3 mujeres lo padece; con frecuencia la violencia se da a mujeres jóvenes de temprana edad que suelen oscilar alrededor de los 15 y 24 años; así mismo un estimado de 641 millones de mujeres que recibieron la agresión, fue por parte de sus parejas o familiares.

En el Perú las estadísticas son igual de alarmantes, durante los años comprendidos entre el 2015 y 2019, 5 de cada 10 mujeres sufrió violencia provocado por sus parejas (Sorenson et al., 2021); de las cuales el 52,8\% sufrió violencia psicológica y verbal, el 29,5\% experimento violencia física y un 7,1\% recibió agresión sexual (CEIC, 2021).

Ante los índices alarmantes los gobiernos han desarrollado programas, promulgado leyes, establecido sistemas nacionales, entre otras medidas, con la finalidad de la prevención y erradicación de la violencia contra las mujeres. A este conjunto de acciones; que ha mediado el estado en el afán de poder brindar garantías constitucionales de los derechos fundamentales de las personas, como el derecho a la paz social de una vida digna dentro del marco de protección (Candia, 2017); se le denomina políticas públicas.

Las políticas públicas son la manifestación de objetivos y finalidades que surge por pautas de acción colectiva; en lo político, el interés se centra en realizar acciones frente a los asuntos del colectivo social, entre los intereses suelen estar catalogados como económico, político, cultural o social (Pacheco, 2019). En ese sentido el papel principal lo toma el estado, quien determina y establece dirección, seguido de los demás participantes adecuadamente estructurados por los distintos territorios para gestar las políticas e informarse adecuadamente y fijarse un rumbo en base a la situación real y particular de cada una de ellas.

Investigaciones relacionas a la aplicación de políticas públicas, como la de Somocursio (2019), señalan que las mujeres que han sufrido violencia no tienen respaldo de una red familiar de apoyo, nublan la razón por lo que no son conscientes de la situación de riesgos que viven; es en estas situaciones que el estado pretende brindar apoyo mediante ,medidas 
de protección, pero no suelen ser funcionales a largo plazo por lo que existe un alto riesgo de que la mujer vuelva a su agresor y ser nuevamente violentada, que en diversas situaciones fatales termina en feminicidio.

Otras investigaciones en temas de salud que tratan las condiciones internas y de convivencia que viven las mujeres y los factores que provocan violencia contra la mujer buscan generar resultados que describan las dimensiones y características propias de la generación de violencia familiar con el propósito de facilitar el desarrollo de políticas públicas y brindar un mejor entendimiento del panorama para atacar directamente a las causas y encontrar ,mejores medidas para contrarrestar la violencia en las mujeres.

En el Perú, la creación de la ley 30364, Ley para prevenir, sancionar y erradicar la violencia contra las mujeres y los integrantes del grupo familiar y su reglamento, ha creado un Sistema Nacional para la "Prevención, Sanción y Erradicación de la Violencia contra las Mujeres e Integrantes del Grupo Familiar", por el cual se pretende asegurar el cumplimiento de las políticas públicas, para ello requieren la participación integra de todas las entidades gubernamentales; por tal razón se ha creado instancias de concertación para la gestión e implementación de políticas públicas para la prevención, atención y sanción de la violencia contra la mujer, en conformidad con las políticas nacionales, y es sustancial la participación de la ciudadanía, impulsados por los gobiernos regionales, provinciales y distritales (Observatorio Nacional de la violencia contra las Mujeres y los Integrantes del Grupo Familiar, 2018).

Con base en lo expuesto, se plantea el objetivo de describir y caracterizar las teorías referentes a las políticas públicas y la lucha contra la violencia a la mujer, así como describir la violencia contra la mujer como un problema social; y describir como la mujer ha quebrantado paradigmas culturales y su rol ante la sociedad.

\section{ESTRATEGIAS METODOLÓGICAS O MATERIALES Y MÉTODOS}

Para la realización del artículo, el enfoque metodológico que se desarrolla es el descriptivo, puesto que se describen los fenómenos relacionados a la aplicación de políticas públicas a favor de la lucha contra la violencia de la mujer en Latinoamérica. Las fuentes básicas son recabadas de artículos indexados en Scielo y Scopus.

Se emplearon técnicas de recolección de datos y análisis de información cualitativa; se empleó el método de análisis documental, se realizó el análisis de las fuentes informativas con la herramienta de recolección de datos denominados "documentos, registros, 
materiales y artefactos"; que básicamente se convierten en fuentes valiosas para la recolección de datos cualitativos, las cuales permiten al investigador conocer los antecedentes, las vivencias o situaciones particulares que se producen en un entorno $\mathrm{u}$ ambiente a través de la utilización de documentación de cualquier tipo, así como archivos, entre otros. (Hernández y Mendoza, 2018).

Respecto al análisis y recolección de datos, se sigue una línea secuencial del conjunto de procesos comprendidos como; la revisión de todos los datos obtenidos, la determinación de los criterios de organización de los datos, la preparación de los datos para el análisis, el descubrimiento de unidades de análisis, la codificación de las unidades en un primer nivel, posteriormente prosigue la descripción de categorías emergentes así como la codificación axial de las mismas, culminando con la generación de descripciones o manifestación de interconexiones entre las categorías y los temas de interés para la investigación (Hernández y Mendoza, 2018).

\section{DESARROLLO}

\subsection{Aproximaciones teóricas}

Vigano y Laffin (2019) y Tavares et al. (2017), afirman que la violencia a la mujer surge como una doctrina de crianza de años, alimentados por la "familia, la iglesia y la escuela", tal ha sido el impacto que la doctrina, que la "violencia de la mujer" se interpretó como "educación" ante un mero esfuerzo por controlar y tener a merced a las mujeres y disciplinarlas por supuestamente, no respetar a sus hombres, es decir a su padre, hermanos, esposo.

Este contexto se dio con mayor frecuencia en épocas pasadas pero que aún persisten en el tiempo e incluso llega a ser fatal para la víctima, este tipo de acción son trasmitidas de generación en generación a modo de cultura, donde predomina una estructura jerárquica donde la mujer es después del hombre y debe someterse a él, a su voluntad y su poder (Tavares et al., 2017). Sin embargo, el tiempo es un factor que evoluciona y trasciende los fundamentos de las épocas pasadas y los trasforma a conocimiento y nuevas experiencias, por ello las mujeres empezaron a cuestionar el poder que se atribuyó el hombre y que puso a la mujer por debajo de él con la premisa de que bajo ninguna circunstancia debía pasar o faltar a dicho poder. Las feministas, termino otorgado a mujeres que luchan por la igualdad y el reconocimiento de la mujer con los mismos derechos que un hombre; lograron avances y el incentivo de mover mayores masas de 
multitud de mujeres aclamando por los mismos derechos e igualdad de género, pero claro ante una acción trasciende una reacción que lo provocaron de una parte los hombres como individuos y de otra parte el estado y la sociedad.

El estado; promovido por las masas y presión de las mujeres e instituciones que compartían el ideal de igualdad de género; planteo principios constitucionales para el fortalecimiento de la igualdad de género, indistintamente de la raza, edad, nacionalidad o estatus económico, dicho de otro modo, el estado centro las políticas públicas en la lucha contra la violencia a la mujer.

Según Calife et al. (2018) y Vigano y Laffin (2019), las políticas públicas como respuesta a la violencia contra la mujer, es un conjunto de normas y promulgados que reconoce como factor de violencia el género y la vulnerabilidad de los derechos humanos de las mujeres.

\subsection{Violencia contra la mujer como problema social}

La violencia contra la mujer no discrimina, clase social, país, cultura, por ello las manifestaciones de agresión ya sea psicológica, sexual, física o económica, se da en todo el mundo; y es esta razón fundamental que, en 1993, la asamblea general de las naciones unidas emitió la declaración de hacer efectiva la supresión de la violencia contra la mujer y en 1996 la Organización Mundial de Salud declaró la violencia contra la mujer, como prioridad pública (Vargas, 2017; Chávez, 2018; Sánchez , 2018 y Pearson et al., 2021); pero esta declaración tenía un trasfondo y sustento científico; un solo elemento como la violencia a la mujer desencadenaba en otros efectos y consecuencias perjudiciales para la sociedad. Vargas (2017) señala que "la violencia contra la mujer puede ocasionar efectos en su salud física y mental, así como, en su relación con la familia, el ámbito relacional y la comunidad" (p. 52); uno de las consecuencias que afecta a las mujeres violentadas y su entorno son efectos sobre la salud mental.

Vargas (2017) establece que la violencia que se ejerce sobre la mujer, a largo plazo se manifiesta de numerosas formas, como la depresión, baja autoestima, estrés postraumático incluido los intentos de suicidio, todo ello engloba problemas mentales. Vargas señala que "La prevalencia promedio encontrada fue: trastorno de stress post traumático $(63,8 \%)$, trastorno depresivo $(47,6 \%)$, suicidios $(17,9 \%)$, abuso o dependencia de alcohol (18,5\%) y abuso o dependencia desustancias psicoactivas (8,9\%)" (p. 52). En Perú los índices también se encuentran en los márgenes y tendencias presentadas por otras 
investigaciones con el adicional de que las mujeres violentadas también manifiestas estados anímicos bajos, señalando que siempre se encuentran tristes y preocupadas; esta sensación, que provoca la violencia en la mujer no solo recae en ella, sino en sus propios hijos, ya sea que hayan visto y sufrido violencia junto con su madre o no.

La familia es el pilar de la sociedad; un hogar cálido, afectivo, comprensivo contribuye al desarrollo de los niños y los adolescentes; la influencia que se ejerce es multifactorial, es decir corresponde a otros factores más, pero el factor clave para su desarrollo es el hogar; una constante muestra de desequilibrio familiar afecta al niño o adolescente y se manifiesta por problemas en su salud mental como depresión infantil, agresividad, baja autoestima y otros factores relacionados (Vargas, 2017); estas consecuencias de problemas en la salud mental suelen ser clasificados como consecuencias físicas, emocionales, cognitivos y problemas de conducta. Así mismo en Perú uno de los índices que alarman al estado y la población son las tasas de incremento de suicidios de adolescente entre las edades de 15 y 24 años; y que representa la tercera causa de muerte de adolescentes, otra manifestación de afección en adolescentes son las huidas del hogar y manifestación de conductas delincuenciales. Todo el manojo que conlleva la violencia contra mujer y como afecta a sus hijos y su entorno; también, están interrelacionados a otros problemas de orden público; y que mejor, que combatir la principal fuente que altera el orden y desencadena las fatales consecuencias en las mujeres violentadas y su entorno, por ello el estado busca la aplicación de políticas públicas para mitigar y reducir en gran medida las consecuencias producto de la violencia de la mujer.

Vélez y Palacios (2017) señalan que el problema de la violencia contra la mujer deber ser tratado con aspectos y elementos íntegros, las investigadoras consideran que al tratar la violencia contra la mujer de forma íntegra se puede crear mejores y eficientes mecanismos que contribuyan a la lucha; entre estos mecanismos se encuentran las políticas públicas; recordando que entre las barreras aplicativas y de funcionalidad, se obtiene la falta de identificación de los focos y elementos que se encuentran inmersos en los actos de violencia contra las mujeres así como la falta de integración de los elementos del estado para producir información relevante y contribuir a la elaboración de nuevas políticas públicas. Este abordaje integral que se sugiere consta de análisis de factores políticos, culturales, económicos y de salud. 


\subsection{La lucha contra la violencia a la mujer en Latinoamérica}

La violencia contra la mujer ha sido un problema proveniente de años anteriores, previamente se realizaron pesquisas e investigaciones que daban gritos de alarma y alerta de este tipo de violencia, así como sus graves consecuencias (Feminicidio), pero aun así muchos países en el mundo no consideraron a la violencia contra la mujer como un problema social (Signorelli et al., 2018).

En américa Latina, se llevó acabo toda una odisea para que el estado de cada país pueda dar la atención debida a la violencia contra la mujer. Según Damián y Flores (2018), en México, el estado por décadas hizo caso omiso y no atribuyo su atención a la violencia contra la mujer como un problema de interés social, hasta lo sucedido en los años de 2001, donde la corte interamericana de Derechos Humanos sentencio al país e hizo atribuible y responsable de la muerte de 3 mujeres en el año de 1995, “se le condenó por violar sus derechos humanos y por incumplir sus deberes de protección por los hechos vinculados a las tres víctimas y sus familias" (Damián y Flores, 2018, p. 35). Este hito en la historia marco al gobierno y por fin se dio la atención interesada a este problema latente en México, próximamente durante el pasar de los años el país tuvo una transición accidentada en la creación de políticas públicas para atacar a los índices altos de violencia; sin embargo, no se dejó de hacer esfuerzos; como resultado se estima que le tomo al paisa alrededor de 2 años para realizar el proceso de alerta de violencia de género, como resultado se obtuvo a un 37,5\% de organismos que aceptaron el proceso de declaración de alerta y asimilaron los nuevos lineamientos en sus funciones y así contribuir con acciones; dentro de estas se encuentran organismos institucionales del estado, denominadas instituciones federativas; sin embargo también hubo instituciones que no la aceptaron las cuales ascendieron a un total de $21,8 \%$.

En Chile, la manifestación de las políticas públicas se dio a inicios de los años 90, con la creación de la ley de Violencia Intrafamiliar en 1994, por el cual se facultaba a la violencia intrafamiliar como delito; posteriormente en los años 2000 se produjeron nuevas inserciones para el mejoramiento de la ley. En ese sentido se produjeron situaciones favorables que coadyuvaron a la trasformación legal y pública para la lucha contra la violencia a la mujer, uno de los sectores que ayudo a la conformación de las políticas y la legislación fue la reforma judicial que atravesaba en los año 2004, donde se creó el tribunal de la familia y penalmente "se modificó todo el proceso de un modelo inquisitivo 
a un acusatorio, poniéndose en marcha nuevos tribunales: Tribunales de Garantía y el Tribunal oral en lo Penal" (Miranda y Gac, 2020, p. 162). Miranda y Gac (2020), señala que las reformas e instauraciones de nuevas formas de estructura para la lucha contra la violencia a la mujer, tuvo varios altibajos, primordialmente al momento de entablar adecuadas políticas públicas, uno de las principales dificultades, es sin duda los agentes $\mathrm{u}$ actores que disciernen y plantean las políticas públicas, la complejidad en el entendimiento de las circunstancias y vivencias de los actores dificulta la identificación precisa de la causa y foco de violencia contra la mujer que pueda ser implementado y logre los objetivos iniciales de la implementación de las políticas públicas. Todo conlleva procesos largos, complejos, que requieren de conocimientos, de experiencias, de la propia formación cultural y la educación, “el trabajo se traduce entonces en la toma de notas, pero también en conversaciones informales y en la posibilidad de interactuar con un abanico amplio de actores durante el proceso de implementación” (Miranda y Gac, 2020, p. 163).

Otro país en la lucha contra la violencia a la mujer fue Bolivia, según Chávez (2018), en Bolivia aún existe un vacío respecto a las bases analíticas al momento de la aplicación de políticas públicas; su fundamento se base que en terminos legales no se está muy bien definida la violencia contra la mujer, las interpretaciones son amplias y parten de diferentes juicios y criterios, además existe una brecha enorme que imposibilita el análisis de datos y de los casos presentados por denuncia ante agresiones, pues existen brechas entre la cantidad de denuncias presentadas por agresión a la mujer y los casos efectivos que se convierten en sentencia condenatoria. Al no contar con información verificada y aproximaciones confiables para la determinación de las principales causas de violencia y la falta de la comprensión y entendimiento del entorno, son pocas las bases que el estado boliviano tiene para plantear política publicas eficientes y precisas. Por otro lado, los datos que si tienen cabida para el análisis, son aquellos que fueron denunciados y tipificados como feminicidio, de las cuales se afirma que existe una tendencia creciente en los últimos años, sin ir muy lejos durante el 2017 se pasó de 8 casos de feminicidio a 109 casos, esto debido al tratamiento y aprobación de la ley $\mathrm{N}^{\circ} 348$ del año 2014.

En Perú, según el CEIC (2021), la normatividad legal para erradicar la violencia contra las mujeres fue promulgado a través de la ley $\mathrm{N}^{\circ} 26260$ en 1993; posteriormente se promulgaron nuevas leyes en búsqueda de la disminución y erradicación de la violencia 
contra la mujer, facilitando y dotando a instancias públicas para el tratamiento y el cumplimiento del debido proceso requerido para la consecución de los objetivos instaurados por las leyes. CEIC (2021) señala las siguientes leyes en línea cronológica:

- Ley $\mathrm{N}^{\circ}$ 30364: Ley orientada a prevenir, sancionar y erradicar la violencia contra las mujeres y los integrantes del grupo familiar (2015)

- Decreto Legislativo $\mathrm{N}^{\circ}$ 1323: Que Fortalece La Lucha Contra El Feminicidio, La Violencia Familiar Y La Violencia De Género (2017)

- Decreto supremo 008-2019-MIMP, que aprueba la Política Nacional de Igualdad de Género (2019)

Uno de los índices actuales de Perú, que respecta a la violencia de la mujer, son las tasas de feminicidio, uno de los actos más crueles y violentos que acaba con la vida de las mujeres. Según el CEIC (2021) los ultimo 5 años comprendidos entre 2015 - 2019, muestran las cifras de feminicidios como se ve en la tabla 1, por cada año respectivamente, así como una clara tendencia de crecimiento; sin embargo, para el 2019 se mantuvo en su margen respeto al año anterior y tuvo un ligero descenso.

\section{Tabla 1}

Índices de feminicidio en Perú - 2015 al 2019

\begin{tabular}{lcccc}
\hline Año & $\begin{array}{c}\text { Victimas por } \\
\text { feminicidio 1/ }\end{array}$ & $\begin{array}{c}\text { Tasa de feminicidio } \\
\text { por cada 100 mil } \\
\text { mujeres 2/ }\end{array}$ & $\begin{array}{c}\text { Variación } \\
\text { \% }\end{array}$ & $\begin{array}{c}\text { Variación } \\
\text { acumulada } \\
\text { \% }\end{array}$ \\
\hline 2015 & 84 & 0,5 & - & - \\
2016 & 106 & 0,7 & 26,2 & 26.2 \\
2017 & 131 & 0,8 & 23,6 & 49.8 \\
2018 & 150 & 0,9 & 14,5 & 64.3 \\
2019 & 148 & 0,9 & $-1, .3$ & 63.0 \\
\hline
\end{tabular}

Nota. Tomado de "Perú: Feminicidio y Violencia contra la mujer 2015-2019” (p. 18), por CEIC, 2021.

Ahora bien, se muestra también los índices o tasas de feminicidio según departamentos del país en un periodo de tiempo de 2015 al 2019. 
Figura 1. Perú: Tasas de feminicidio según departamento - 2015 al 2017
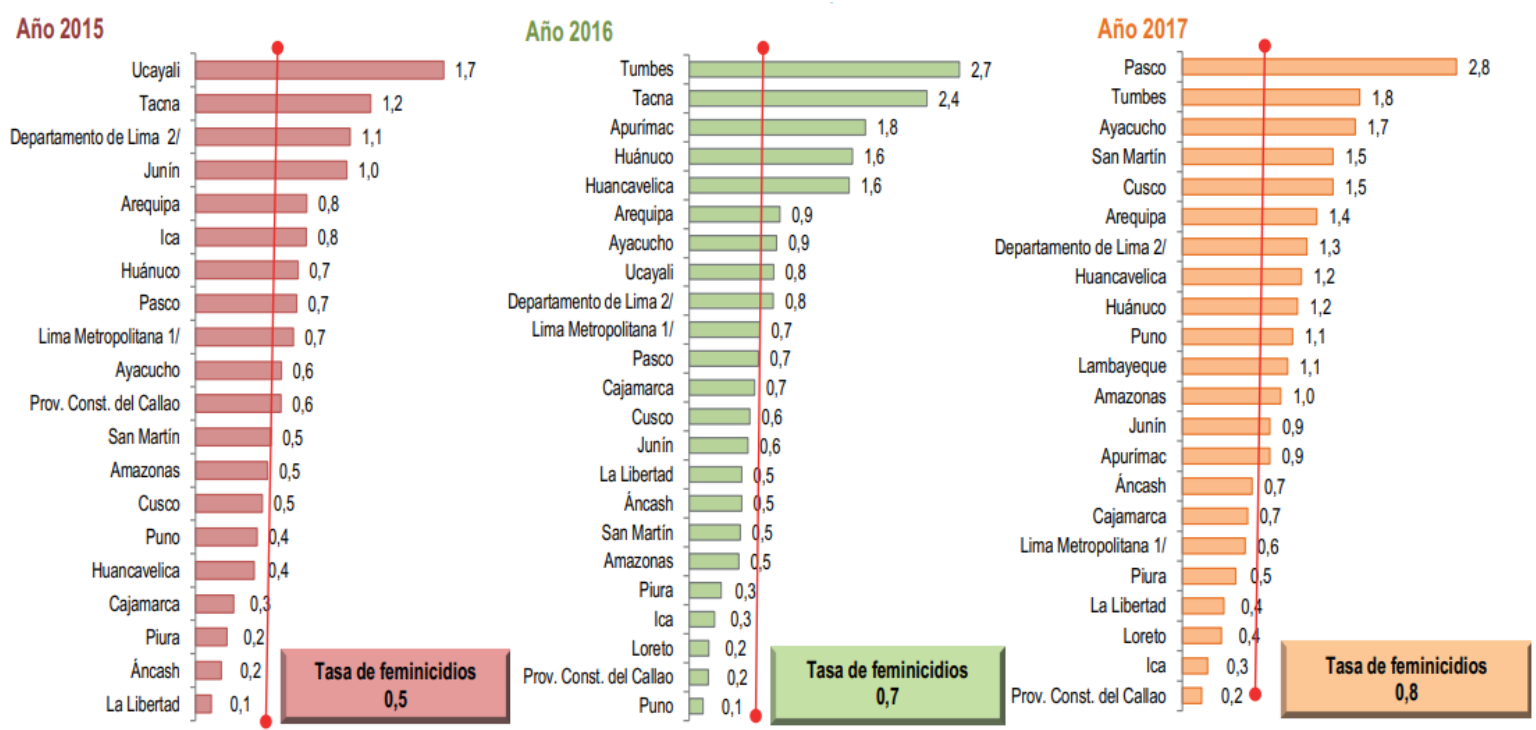

Nota. Tomado de "Perú: Feminicidio y Violencia contra la mujer 2015-2017” (p. 19), por CEIC, 2021.

Figura 2. Perú: Tasas de feminicidio según departamento - 2018 al 2019
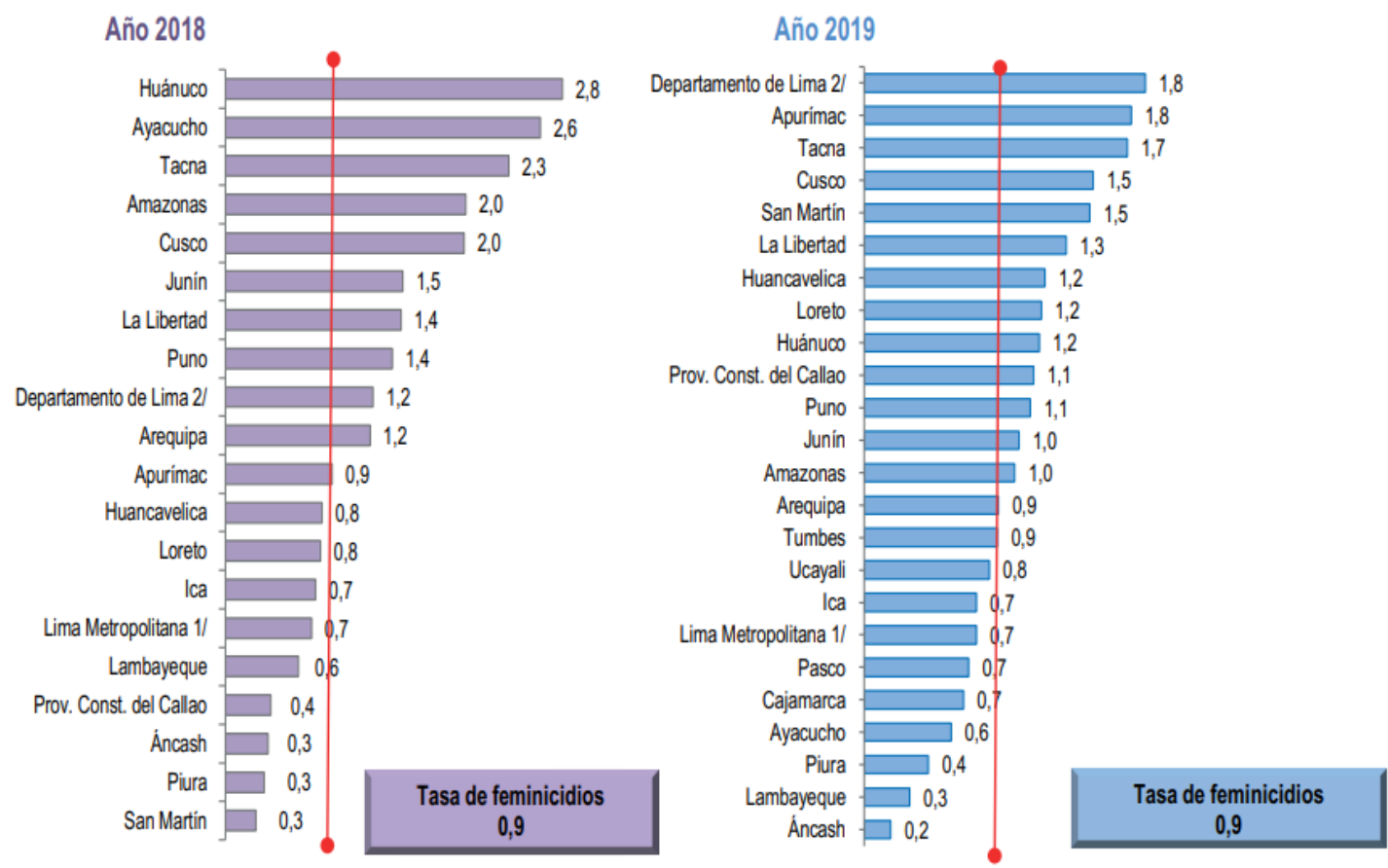

Nota. Tomado de "Perú: Feminicidio y Violencia contra la mujer 2015-2019” (p. 19), por CEIC, 2021. 
Según el CEIC (2021) en base a los índices mostrados en las gráficas y tablas, el indicador de feminicidio es el indicador con mayor precisión y exactitud que permite realizar políticas públicas en base al seguimiento, la evaluación y la comparación de las tasas; además se toma en cuenta las condiciones sociodemográficas particulares de las víctimas, así como los presuntos feminicidas.

\subsection{La mujer y su participación en la política}

Las mujeres tienen una participación muy limitada en las diferentes líneas y sectores políticos funcionales de sus países, normalmente los puestos políticos son cubiertos por hombres en su mayoría, a esta representación se le denomina cuota. En Europa la cuota de representación de la mujer en puestos y cargos públicos en el año 2016 solo fue representada por un 26\% que formaron parte de los miembros de la Unión Europea; ante tal representación de la cuota de mujeres en la política despertó inquietud en la mayoría de los países europeos, por lo que este suceso represento un nuevo punto en la agenda política de numerosas naciones. Si bien en varios países se tomó la iniciativa para incentivar el incremento de la participación de la mujer en la política; los altos cargos políticos, lógicamente precedidos por hombres, manifestaron conductas y opiniones que a su criterio no consideraban correcto que mujeres formen parte del staff político. La explicación más cercana de las razones del porque existen dos posturas frente a que la mujer incremente su participación en la política, lo brinda Fernández y Valiente (2021), los cuales recabaron previas investigaciones que esclarecen el panorama y se explica que las dos posturas responden a 4 categorías principales, las cuales constan de factores sociodemográfico, económico, político y cultural.

\subsubsection{Explicación sociodemográfica.}

Lamentablemente aún existen prejuicios de género, ha sido una dura lucha para derribar paradigmas de épocas pasadas; sin embargo, aún persiste los prejuicios y por ello las personas que apoyan a la oposición de que la mujer participe en la política; consideran que las mujeres no deberían de estar en cargo de funcionarios ya sea público o privado, y que factores como la edad, el estado civil, el número de hijos que tienen, la remuneración que percibe y el nivel de educación, son factores de riesgo que de ser favorables incentivan a las mujeres a crecer y por ello amenaza el estatus de los funcionarios (Redding et al., 2017). 


\subsubsection{Explicación económica}

La literatura manifiesta que la economía es una de las explicaciones más relevantes del porqué de la oposición; el desarrollo económico influye en la cultura de un determinado país, así como en los ideales, uno de los temas de mayor afección son los ideales respecto al género, el papel del hombre en el hogar, el empleo y la política. Generalmente países con índices favorables de desarrollo y crecimiento económico, manifiestan altos índices de participación de la mujer en la política, pero en los países no tan desarrollados como países pertenecientes a Latinoamérica o sectores económicos como la agraria, persisten ideas tradicionales debido al riesgo e incertidumbre de calamidades que los afectan más que a otros, por ello el hombre toma el papel de proveedor y la mujer toma el papel de ama de casa, cuidar a los hijos, atender a su pareja, que notoriamente no tiene nada que ver con que la mujer pueda realizar otras actividades.

\subsubsection{Explicación política}

La principal explicación que surge del porque las mujeres reciben poco apoyo para la participación en temas políticos, son los propios intereses políticos de los ejecutivos. Por otro lado, la ideología de centro izquierda son los que manifiestan interés por incentivar e impulsar a las mujeres en temas políticos, además depende del nivel de conocimiento de los interesados y su apego al interés político pues a causa de ello ganan experiencia, conocimientos y más que nadie reconoce la carencia de la participación femenina en la política. Al lograr la incorporación de las mujeres en los parlamentos y otras instancias políticas, también se establecen y crean nuevas políticas de igualdad de género y otras relacionadas a la mujer y su participación activa en los diferentes sectores.

\subsubsection{Explicación cultural}

Este tipo de explicación se enfoca en el arraigo cultural de las creencias y formas y estilos de vida. Aún persiste la creencia y la manifestación de los roles de la mujer en la sociedad (familia, empleo, política) (Redding et al., 2017); sin embargo, también se han manifestado nuevas visiones conjuntas e igualitarias que fomentan la igualdad de oportunidades, por lo que son más personas y organismos que apoyan la participación de la mujer en la política.

Ya entablado las razones y el escenario de la mujer en la política es preciso hacer hincapié del porque es importante que la mujer tenga más participación en la política, y responde a que como mujeres se conocen las situaciones difíciles que atraviesan a diario y la 
constante amenaza a la integridad y salud de la mujer, por ello como se mencionó anteriormente, incentivar la participación de la mujer en la política, permite la generación de igualdad de oportunidades y también se crean políticas de gobernanza que contribuyen y favorecen la situación de las mujeres en la sociedad. Existe una clara tendencia que, a mayor participación de las mujeres en la política, también se incrementa el mayor número de legislación y políticas publicas emitidas a favor de la mujer (Fernández y Valiente, 2021).

\subsection{Políticas públicas en los gobiernos y sus barreras aplicativas}

Según Calife et al. (2018) y Vigano y Laffin (2019), a nivel mundial la violencia contra las mujeres llega a cifras alarmantes, los casos que son registrados son severos e incluso repetitivos en los mismos individuos. Los principales agentes que se encuentran en primera mano y conocen la situación de agravio con las que llegan las mujeres agredidas de forma física, psicológica, son el personal médico y de salud de los establecimientos donde son atendidas (Doares et al., 2017), este hecho no pasa por desapercibido, por ello los gobiernos locales, son los primeros que al identificar que los agentes principales de contacto directo con mujeres violentadas son el personal médico de los hospitales o postas médicas, han dispuesto conjuntos de políticas y procedimientos que coadyuven a la lucha contra la violencia de la mujer; sin embargo, como lo señala Calife et al (2018), existe una gran brecha entre las disposiciones y la practica social de ellas. Una de las barreras aplicativas frecuentes a la hora de instaurar políticas públicas radica en los agentes que evalúan, monitorean las formas, condiciones y posibilidades de la gestación de nuevas políticas públicas; generalmente para atacar de frente a la problemática de la violencia contra la mujer es necesario identificar los principales focos de manifestación de violencia o en su defecto los principales momentos en los cuales se identifica el agravio; una vez hecha la identificación de estos focos principales es necesario el entendimiento del contexto y la colaboración conjunta de los involucrados, para el desarrollo y creación de una nueva política pública. Los agentes de salud son la base de primera mano que brindan el soporte para el entendimiento de la violencia que sufrieron las víctimas; sin embargo, las instituciones de salud no colaboran y limitan sus acciones a esfuerzos escasos por lo que, sin la colaboración de estos agentes, el trabajo se limita y no se puede atacar directa y efectivamente a los factores de riesgo de manifestación de violencia y por ende en los esfuerzos por manifestar acción y posturas del gobierno se elaboran políticas poco 
eficientes y las cuales no logran su objetivo. Para que una política pública sea ejecutada a cabalidad requiere sustancialmente la colaboración de todos los participantes, a modo de ejemplo se requeriría que la agraviada, manifieste las circunstancias y el entorno en las que se manifestó la violencia; por otro lado, los puestos de salud deben de aplicar los procedimientos establecidos ante un caso de violencia registrada, tomando en cuenta aspectos de salud física y psicológica, así como cumplir con el debido proceso de ejecución de las políticas impuestas por el gobierno, y también es necesaria la participación del gobierno no solo en el dictamen de las políticas sino de velar por el cumplimiento de ellas, con mecanismos de evaluación y supervisión (Doares et al., 2017).

\subsection{Participación política de instancias regionales y locales}

Uno de los problemas y barreras que limitan la aplicación y la creación de nuevas políticas públicas son los propios actores, se ha señalado que es necesaria la colaboración y participación de las instancias y organismos a los que compete, fomentando la supervisión y evaluación constante. Entre los factores de fracaso en el cumplimiento del objetivo de las políticas públicas es la falta de compromiso para llevar a cabo tareas y procesos respecto a la aplicación y ejecución de dichas políticas.

Chávez (2018) señala que la participación: “depende directamente de la forma en que estén organizadas las estructuras políticas, la forma de interacción de los sujetos políticos y de las prácticas que dan forma a procesos que ponen en juego el poder político y lo que se entiende como espacio público" (p. 27). Entonces, cuando se señala que es necesaria la participación política se refiere a la interacción e intervención de los organismos en el proceso de la construcción de las políticas públicas, esto como contraprestación al poder que ejercer los representantes políticos.

La actualidad es cambiante, y el tiempo trae consigo nuevos esquemas, paradigmas, y se gestan nuevos contextos, tal es el caso de la pandemia por el Covid-19 a nivel mundial, un hecho que sin dudas incremento las tasas e índices de violencia contra la mujer y los niños (Sorenson et al., 2021); Pearson et al (2021), manifiesta que la ONU la OMS y otras agencias, publicaron políticas aplicables para los gobiernos del mundo, con el propósito de poder contrarrestar la consecuencia del incremento de las tasas de violencia en los periodos de cuarentena y la pandemia por el Covid-19; señalando que, la prevención, la mitigación y respuesta ante la violencia contra la mujer y los niños es de entera responsabilidad de los gobiernos y los formuladores de políticas, por ello la ONU, la OMS 
y otros, emplear métodos de gestión y difusión de información al público sobre el riesgo inherente de la violencia contra la mujer y más aún por la cuarentena por el Covid-19.

Finalmente, uno de los puntos más resaltantes en la participación integral de las instancias del gobierno en la elaboración de las políticas públicas en base a los datos obtenidos y los datos integradores; es la estructura orgánica que siguen los actores gubernamentales; según la ley 30364, en el Perú, se creó el "Sistema Nacional para la Prevención, Sanción y Erradicación de la Violencia contra las Mujeres e Integrantes del Grupo Familiar", el cual es un sistema exclusivamente orientado a velar por el cumplimiento de políticas publicas relacionada a su tema referente. Por tal motivo realiza las coordinaciones con los diferentes niveles de gobiernos existente. La estructura de conformación e instancias se muestran en la figura siguiente:

\section{Figura 3. Instancias de concertación - Niveles de Gobierno}

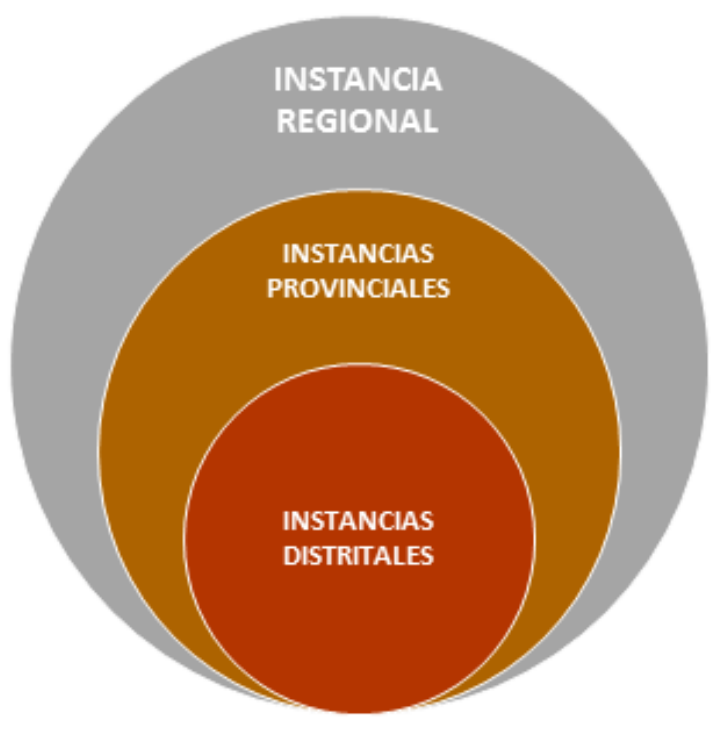

Nota. Tomado de "Rol de los gobiernos locales y regionales para la prevención y erradicación de la violencia contra las mujeres e integrantes del grupo familiar", por el Observatorio Nacional de la violencia contra las Mujeres y los Integrantes del Grupo Familiar CEIC, 2021.

"Las instancias de concertación constituyen los espacios formales de propuesta y evaluación de políticas públicas destinadas a la erradicación de la violencia contra las mujeres e integrantes del grupo familiar. Distritales" (Observatorio Nacional de la violencia contra las Mujeres y los Integrantes del Grupo Familiar, 2018). 
No cabe duda que en el Perú se requiere de la participación activa de los niveles de gobierno, así como los datos e información de la situación de la violencia hacia la mujer, para tomar acción y elaborar nuevas políticas públicas, por ser considerado como un problema social que debe ser atendido en el momento, de forma eficiente, sobre todo dando el esfuerzo necesario y la facilidad en la aplicación de los mecanismos para la aplicación de las políticas con la participación activa de los actores intervinientes, y la clara idea de unificar e integrar los factores posibles que provoquen focos de violencia contra la mujer.

\section{CONCLUSIÓN O CONSIDERACIONES FINALES}

- La violencia contra la mujer desde épocas pasadas se aceptó como una forma de educación, en la que se había establecido que una mujer no podía estar por encima del hombre y no debería de "faltar el respeto", tal fue el arraigo de la creencia que fue transmitido de generación en generación como una especie de cultura. Sin embargo, las feministas, consideradas mujeres que luchan por la igualdad de género, con el tiempo rompieron paradigmas y comenzó la época de lucha por los derechos y gozo a las mismas oportunidades que los hombres.

- La implementación de políticas públicas para mitigar la violencia contra la mujer ha sido un proceso con altibajos, los países en Latinoamérica como México, Bolivia, Chile y Perú han tenido dificultades a la hora de determinar políticas públicas que realmente contribuyan a la disminución de tasas de los índices de violencia; sin embargo, el transcurso del tiempo ha permitido la implementación y mejora de los procesos para la creación de políticas públicas, aun se presentan barreras que competen al esfuerzo colectivo que se debe ejercer para el logro de los objetivos.

- Las políticas públicas comprenden un proceso complejo de entendimiento, por ello es necesario la aplicación de un modelo integral que contemple factores políticos, culturales, económicos y de salud, con el propósito de erradicar la violencia contra la mujer, es importante que para aplicar políticas públicas todos los actores y participantes colaboren.

- La ONU determino que la violencia contra la mujer es un problema social, por los efectos colaterales que manifiesta la mujer violentada en su núcleo familiar, normalmente quien agrede es un pariente, lo cual afecta psicológicamente a la mujer, lo que le genera afección en su salud, en su estado mental en su condición física ello 
trasciende y afecta a su entorno, básicamente los hijos son los que adicionalmente desarrollan condiciones como baja autoestima, problemas de salud, problemas de conducta, llegando a la desintegración familiar, abandono, suicidio de los hijo y conductas delincuenciales. Por comprometer tantas facetas y ser un factor de riesgo para la desintegración familiar, la violencia a la mujer es considerada como un problema social.

- Finalmente, la mujer a través del tiempo a quebrantado y roto paradigmas arraigados culturalmente, se busca el fomento de la inserción de la mujer en la política, la ocupación de cargos importantes y su propia emancipación, la mujer actual no ve al hombre como un proveedor y ella como una sustentadora del hogar, por el contrario, busca nuevas oportunidades; pero aún existe muchos obstáculos por cruzar y uno de ellos es la violencia.

\section{LISTA DE REFERENCIAS}

Comité Estadístico Interinstitucional de la Criminalidad. (2021). Perú: Feminicidio y Violencia. https://oig.cepal.org/es/documentos/peru-feminicidio-violencia-lamujer-2015-2019

Organización Mundial de Salud. (2021). La violencia contra la mujer es omnipresente y devastadora: la sufren una de cada tres mujeres. https://www.who.int/es/news/item/09-03-2021-devastatingly-pervasive-1-in-3women-globally-experience-violence

Signorelli, M., Taft, A. y Gomes, P. (2018). Domestic violence against women, public policies and community health workers in Brazilian Primary Health Care. Ciência \& Saúde Coletiva, 23(1), 93-102. https://doi.org/10.1590/141381232018231.16562015

Sorenson, S., Sinko,L. y Berk, R. (2021). The Endemic Amid the Pandemic: Seeking Help for Violence Against Women in the Initial Phases of COVID-19. Journal of Interpersonal $\quad$ Violence, $\quad 36(9-10), \quad 4899-4915$. https://journals.sagepub.com/doi/full/10.1177/0886260521997946

Candia, K. (2017). Incongruencia en la Aplicación de Principios Constitucionales en La Ley 30364 - Violencia Contra la Mujer y Grupo Familiar. Revista Científica Investigación Andina, $\quad$ 77(2), 76-83. http://repositorio.uancv.edu.pe/handle/UANCV/2798 
Pacheco, L. (2019). Las políticas públicas, retos y escenarios cambiantes hoy. Investigaciones Sociales, 22(40), 291-304.

https://doi.org/10.15381/is.v22i40.15927

Somocurcio, N. (2019). Protección jurídica de la mujer que denuncia violencia en el ámbito familiar. La Vida \& la Historia, 6(1), 41-50.

https://doi.org/10.33326/26176041.2019.9.792

Observatorio Nacional de la violencia contra las Mujeres y los Integrantes del Grupo Familiar. (2018). Rol de los gobiernos locales y regionales para la prevención y erradicación de la violencia contra las mujeres e integrantes del grupo familiar. https://cutt.ly/kTVcjOl

Hernández, R. y Mendoza, C. (2018). Metodología de la investigación: las rutas: cuantitativa, cualitativa y mixta. McGraw-Hill Interamericana Editores. http://repositorio.uasb.edu.bo:8080/handle/54000/1292

Vigano, D. y Laffin, M. (2019). [Women, public policies and fight against gender violence] [Mulheres, políticas públicas e combate à violência de gênero]. Historia (Brazil), 38, 1-18. https://doi.org/10.1590/1980-4369e2019054

Tavares, K., De Toledo, R., Do Nascimento, J., Coelho, H. y Tôrres, E. (2017). Association between domestic violence and women's quality of life. Rev. LatinoAm. Enfermagem, 25, 1-8. https://doi.org/10.1590/1518-8345.1535.2901

Calife, K., Blima, L., Pires, A. (2018). Gestores de saúde e o enfrentamento da violência de gênero contra as mulheres: as políticas públicas e sua implementação em São Paulo, Brasil. Cad. Saúde Pública, 34(8), 1-11. https://doi.org/10.1590/0102$311 \mathrm{X} 00140017$

Vargas, H. (2017). Violencia contra la mujer infligida por su pareja y su relación con la salud mental de los hijos adolescentes. Rev Med Hered, 28(1), 48-58. http://dx.doi.org/10.20453/rmh.v28i1.3074

Chávez, A. (2018). Avance de investigación: ¿La violencia de género es el lente para entender la participación política de las mujeres en Bolivia?. Rev. aportes de la comunicación,

21-32.

http://www.revistasbolivianas.org.bo/scielo.php?pid=S2306-

$86712018000100003 \&$ script=sci_arttext 
Sánchez, T. (2018). Políticas sanitarias e igualdad entre mujeres y hombres. Revista de $\begin{array}{llll}\text { Bioética } & y & \text { Derecho, } & \text { (43), }\end{array}$ http://scielo.isciii.es/scielo.php?script=sci_arttext\&pid=S1886$58872018000200013 \& \operatorname{lng}=\mathrm{es} \& \operatorname{tlng}=\mathrm{es}$.

Pearson, I., Butler, N., Yelgezekova, Z., Nihlén, A., Aguirre, I., Quigg, Z. y Stöckl, H. (2021). Emerging responses implemented to prevent and respond to violence against women and children in WHO European member states during the COVID19 pandemic: a scoping review of online media reports. BMJ Open, (11), 1-11. https://bmjopen.bmj.com/content/11/4/e045872

Vélez, C. y Palacios, L. (2017). Abordar la violencia contra las mujeres desde una perspectiva integral. Revista Ciencias de la Salud, 15(2), 183-187. http://www.scielo.org.co/scielo.php?script=sci_arttext\&pid=S1692$72732017000200183 \& \operatorname{lng}=$ en\&tlng=es.

Fernández, J. y Valiente, C. (2021). Gender quotas and public demand for increasing women's representation in politics: An analysis of 28 European countries. European Political Science Review, 13(3), 351-370. https://cutt.ly/mYdLGm0

Damián, A., Flores, J. (2018). Feminicidios y políticas públicas: declaratorias de alertas de violencia de género en México, 2015-2017. Revista Perspectiva Geográfica 23(2), 33-57. http://www.scielo.org.co/pdf/pgeo/v23n2/0123-3769-pgeo-23-0233.pdf

Miranda, F., Gac, D. (2020). Etnografía y políticas públicas en materia de justicia y violencia contra las mujeres en Chile. Revista Latinoamericana, (55), 159-171. https://scielo.conicyt.cl/pdf/polis/v19n55/0718-6568-polis-19-55-260.pdf

Redding, E., Ruiz-Cantero, M., Fernández-Sáez, J. y Guijarro-Garvi,M. (2017). Gender inequality and violence against women in Spain, 2006-2014: towards a civilized society. Gac Sanit, 31(2), 82-88. https://doi.org/10.1016/j.gaceta.2016.07.025

Soares, L., Pereira, I., Soares, E., Campelo, C., Do Nascimento, A. y Branco, M. (2017). Women's protection public policies: evaluation of health care for victims of sexual violence. Ciênc. saúde colet, 22(5), 1501-1508. https://doi.org/10.1590/1413-81232017225.33272016 
Vigano, D. y Laffin, M. (2019). [Women, public policies and fight against gender violence] [Mulheres, políticas públicas e combate à violência de gênero]. Historia (Brazil), 38, 1-18. https://doi.org/10.1590/1980-4369e2019054

Tavares, K., De Toledo, R., Do Nascimento, J., Coelho, H. y Tôrres, E. (2017). Association between domestic violence and women's quality of life. Rev. LatinoAm. Enfermagem, 25, 1-8. https://doi.org/10.1590/1518-8345.1535.2901

Calife, K., Blima, L., Pires, A. (2018). Gestores de saúde e o enfrentamento da violência de gênero contra as mulheres: as políticas públicas e sua implementação em São Paulo, Brasil. Cad. Saúde Pública, 34(8), 1-11. https://doi.org/10.1590/0102311X00140017

Vargas, H. (2017). Violencia contra la mujer infligida por su pareja y su relación con la salud mental de los hijos adolescentes. Rev Med Hered, 28(1), 48-58. http://dx.doi.org/10.20453/rmh.v28i1.3074

Chávez, A. (2018). Avance de investigación: ¿La violencia de género es el lente para entender la participación política de las mujeres en Bolivia?. Rev. aportes de la comunicación,

21-32. http://www.revistasbolivianas.org.bo/scielo.php?pid=S2306$86712018000100003 \&$ script=sci_arttext

Sánchez, T. (2018). Políticas sanitarias e igualdad entre mujeres y hombres. Revista de Bioética $\quad y \quad$ Derecho, (43), 179-192 http://scielo.isciii.es/scielo.php?script=sci_arttext\&pid=S1886$58872018000200013 \& \operatorname{lng}=$ es\&tlng=es.

Pearson, I., Butler, N., Yelgezekova, Z., Nihlén, A., Aguirre, I., Quigg, Z. y Stöckl, H. (2021). Emerging responses implemented to prevent and respond to violence against women and children in WHO European member states during the COVID19 pandemic: a scoping review of online media reports. BMJ Open, (11), 1-11. https://bmjopen.bmj.com/content/11/4/e045872

Vélez, C. y Palacios, L. (2017). Abordar la violencia contra las mujeres desde una perspectiva integral. Revista Ciencias de la Salud, 15(2), 183-187. http://www.scielo.org.co/scielo.php?script=sci_arttext\&pid=S1692$72732017000200183 \& \operatorname{lng}=$ en\&tlng=es. 
Fernández, J. y Valiente, C. (2021). Gender quotas and public demand for increasing women's representation in politics: An analysis of 28 European countries. European Political Science Review, 13(3), 351-370. https://cutt.ly/mYdLGm0

Damián, A., Flores, J. (2018). Feminicidios y políticas públicas: declaratorias de alertas de violencia de género en México, 2015-2017. Revista Perspectiva Geográfica 23(2), 33-57. http://www.scielo.org.co/pdf/pgeo/v23n2/0123-3769-pgeo-23-0233.pdf 